moraine on the glacier; weighted average of heights of upper and lower ends of a glacier and weighted average of mean height of firn line. It is also necessary to obtain data on glacier area distribution by height, precipitation data, and data on air temperature and cloudiness at meteorological stations.

In addition to defining typical glacial regions within the limits of the glacial area considered and determination of the "average" glacier characteristics listed above, preparation of the computer programme includes (a) definition of precipitation dependence on orography height and calculation of appropriate parameters, (b) reduction (if necessary) of data obtained from the meteorological station nearest to glacial area to a more prolonged range, (c) calculation of a special balance index of accumulation and melting of seasonal precipitation which makes it possible to define maximum excess of seasonal snow line over the "average" glacier terminus in separate years, and (d) computation of total solar radiation occuring in clear days during May to October.

In the course of computation on computer the following operations are realized: (a) determination of an average value of total radiation arriving during months of May to October period given the real cloudiness conditions, (b) calculation of the height of the snow line on the glacier, (c) calculation of quantity and duration of solid atmospheric precipitation which melts between May and October on the glacier surface, (d) estimation of absorbed solar radiation taking account of the differing albedo of glacial surfaces situated above and below the seasonal snow line, (e) calculation of the total melting of glaciers belonging to the given mountain watershed.

Checking of the calculational method has been performed using data from Lednik Tsentralnyy Tuyuksu and Lednik Ayutor-2 where numerous ablation stakes were installed. Values of measured and calculated melting agree satisfactorily.

\title{
SIMULATION OF ICE FLOW USING THE FINITE-ELEMENT METHOD
}

\author{
By T. Q. Nguyen
}

(Montreal Engineering Limited, Montreal, Quebec, Canada)

\author{
and J. J. EMERY
}

(Department of Civil Engineering and Engineering Mechanics, McMaster University, Hamilton, Ontario, Canada)

Abstract. A plane-strain, incremental, initial-strain finite-element analysis, incorporating creep laws appropriate for the ranges of stresses and temperature involved, is used to simulate typical ice-slope flow problems. The flow of a uniform ice slope, assuming the mass is intact and no basal sliding occurs, shows that differences in steady-state velocities can be by orders of magnitude for various creep laws. A non-uniform ice slope and a uniform slope behind a concrete wall are also considered. Time-independent developments of tensile crack and basal shear are investigated by introducing joint elements with appropriate stiffness properties at ice-rock interfaces and tension zones. Then, the simulation model is modified to account for both time-dependent basal sliding (surging) and tensile crack development. In one case, basal shear failure is considered imminent if the shear strain in a thin basal ice layer, that allows wide variation in sliding velocity, exceeds a critical value. For the other case, the basal ice layer is replaced by joint elements and the progressive shear-stress failure mechanism during flow is demonstrated. 